\title{
APOMORFINA
}

\section{UMA ALTERNATIVA NO CONTROLE DAS FLUTUAÇÕES MOTORAS NA DOENÇA DE PARKINSON}

\author{
HENRIOUE B. FERRAZ*, SÔNIA M. C. AZZVEDO-SILVA**, VANDERCI BORGES**, \\ MARIA SHEILA G. ROCHA**, LUIZ AUGUSTO F. ANDRADE***
}

\begin{abstract}
RESUMO - As flutuações motoras (FM) decorrentes do uso prolongado de levodopa são uma das principais complicações do tratamento da doença de Parkinson (DP). A utilização da apomorfina, um potente agonista de receptores dopaminérgicos, associada ao domperidone para bloguear seus efeitos eméticos, surge como uma alternativa para contornar as FM dos parkinsonianos. Para adquirirmos experiência inicial com essa droga, decidimos estudar a ação e os efeitos adversos da apomorfina em um grupo de quatro pacientes do nosso ambulatório com o diagnóstico de DP e com flutuaçāoes de rendimento da levodopa. A apomorfina foi administrada por via subcutânea, sendo obtido o estado "on" em todos os pacientes com doses entre 1,5 e 3 mg por aplicação. A latência para o início do efeito variou de 7 a 30 minutos e a duração da ação de 60 a 85 minutos. O estado "on" produzido com a apomorfina foi indistinguivel do observado com a levodopa, inclusive com a ocorrência de discinesias. Não foram observados efeitos colaterais significativos. Nossa experiência inicial mostra que a apomorfina, em doses relativamente baixas, é uma alternativa eficaz para as FM da DP, com poucos efeitos colaterais.
\end{abstract}

PALAVRAS-CHAVE: doença de Parkinson, tratamento; apomorfina, uso terapêutico; levodopa, efeitos adversos.

Apomorphine: an alternative in the management of motor fluctuations of Parkinson's disease

SUMMARY - Levodopa-induced motor fluctuations (MF) is a disabling complication of Parkinson's disease (PD) and is usually refractory to conventional treatment. Apomorphine, a dopamine agonist with affinity for both $\mathrm{D} 1$ and $\mathrm{D} 2$ receptors, has been emerged as an useful alternative in the managernent of MF of PD. The frequency of nausea and vomiting prevented its use in the past, but the simultaneous administration of domperidone has proved to be able to control these side effects. Although apomorphine has been successfully used to control levodopa-induced MF in other countries, it has not been considered in the management of PD in Brazil. We report here our initial experience with subcutaneous injections of apomorphine combined to oral domperidone. We administered apomorphine in doses ranging from 1.5 to $3 \mathrm{mg}$ in four PD patients with MF of our outpatient clinic. All the doses administered switched the "off" state to a motor response qualitatively similar to what is seen in the "on" phase induced by levodopa, including the occurence of dyskinesia. The latency to tum "on" after apomorphine ranged from 7 to 30 minutes and the duration of the response ranged from 60 to 85 minutes. We observed yawning in all four patients, labial paresthesia in one patient and an inespecific unpleasent sensation in another patient. These side effects were not significant in our four patients. Our data show that the use of apomorphine adds a reliable and effective strategy in the management of MF of PD patients.

KEY WORDS: Parkinson's disease, treatment; apomorphine, therapeutic use; levodopa, adverse effects.

Setor de Investigação em Moléstias Extrapiramidais, Disciplina de Neurologia, Escola Paulista de Medicina: *Doutor em Neurologia, Médico Assistente do Setor; **Pós-Graduando em Neurologia; ***Professor Adjunto-Livre Docente, Chefe do Setor. Aceite: 22-novembro-1994.

Dr. Henrique B. Ferraz - Al. Casa Branca 799 apto 72 - 01408-001 São Paulo SP - Brasil. 
O surgimento da levodopa revolucionou o tratamento da doença de Parkinson no final da década de $60^{7}$. Muitos pacientes seriamente comprometidos readquiriram, com a nova droga, a função motora. O entusiasmo inicial foi grande apesar de alguns problemas terem surgido. Intolerância gastrointestinal foi um dos efeitos colaterais mais observados no início. Além disso, alterações psiquiátricas e hipotensão ortostática foram notadas em alguns pacientes. Com o tratamento prolongado, outros problemas surgiram, em particular as flutuaçōes do rendimento e as discinesias induzidas pela levodopa ${ }^{23}$. Os principais tipos de flutuações motoras (FM) são: a deterioração de final de dose (wearing-off) e as mudanças bruscas e imprevisíveis do estado de mobilidade do paciente (efeito on-off) ${ }^{10}$. As FM são, às vezes, mais incapacitantes que os sintomas da doença, porque os pacientes podem ficar a maior parte do tempo em períodos "off" (acinéticos).

Não há ainda uma explicação definitiva para a ocorrência das FM, mas especula-se que possam ser devidas a alterações na farmacocinética da levodopa, quer por alteração na absorção e transporte, quer por redução da capacidade de estocagern de dopamina pelos neurônios ${ }^{9,24}$. As flutuações imprevisíveis parecem depender menos de mecanismos farmacocinéticos mas, principalmente, de alterações de receptores pós-sinápticos dopaminérgicos ${ }^{2,24}$.

Apesar destas importantes complicações, a levodopa continua sendo a pedra angular do tratamento da doença de Parkinson, porque nenhuma das drogas posteriormente descobertas conseguiu superá-la em eficácia e facilidade de uso. Desse modo, sendo a suspensão da levodopa impossível, na maioria dos pacientes com FM devem-se utilizar estratégias para tentar minimizar estes efeitos adversos.

Recomenda-se, inicialmente, fracionar ao máximo as doses da levodopa, já que níveis plasmáticos mais estáveis estão relacionados a respostas motoras mais sustentadas ${ }^{29}$. $O$ surgimento da levodopa de liberação controlada, pode determinar niveis plasmáticos mais estáveis, beneficiando alguns pacientes. Nesta apresentação são desnecessárias as múltiplas doses no decorrer do dia ${ }^{3}$. Outra medida auxiliar no manejo das FM, é a orientação ao paciente para evitar alimentos protéicos próximo ao horário de tomada da levodopa. Alguns aminoácidos, como a fenilalanina, a leucina e a isoleucina podem interferir no transporte da levodopa plasmática pela barreira hematoencefálica $(\mathrm{BHE})^{25}$. O uso de drogas agonistas dopaminérgicas, em geral associadas às outras medidas citadas, é outra alternativa para o manejo das flutuações. Os agonistas dopaminérgicos mais frequentemente utilizados são os derivados ergolínicos: bromocriptina, pergolida e lisurida. Estes agonistas agem điretamente sobre o receptor pós-sináptico, não dependendo, portanto, da integridade do neurônio dopaminérgico, que está degenerado na doença de Parkinson. Estas drogas podem ser administradas por via oral e permitem, em tese, a estimulação continuada dos receptores dopaminérgicos. Apesar destas vantagens, na prática clínica, grande número de pacientes tem apenas solução parcial do problema, já que a redução dos períodos “off” não é satisfatória. Além disso, estas drogas têm efeitos colaterais proeminentes que são limitantes para um número relativamente grande de pacientes. Estes fatores diminuíram muito o valor potencial dos agonistas dopaminérgicos ${ }^{3}$.

Com essas dificuldades no manejo das FM, passou-se a considerar o uso da apomorfina para alguns pacientes. A apomorfina é um potente agonista de receptores D1 e D2, portanto com ação farmacológica similar à dos derivados ergolínicos ${ }^{12}$. Embora sua ação na doença de Parkinson tenha sido demonstrada quase que simultaneamente à da levodopa, seu efeito emético desestimulou o prosseguimento dos ensaios clínicos ${ }^{5}$. A retomada das pesquisas com a apomorfina se deveu, principalmente, ao controle ineficaz das flutuaçōes de rendimento da levodopa.

Inicialmente, administrou-se a apomorfina por via oral mas, para serem eficazes, as doses tiveram que ser muito elevadas. Todos os pacientes submetidos a estas altas doses de apomorfina (600 a $1400 \mathrm{mg}$ ao dia) apresentaram uremia que, embora reversível, limitava o seu uso ${ }^{6}$. A ministração parenteral necessitava de doses muito menores para serem efetivas (a partir de $1 \mathrm{mg}$ ao dia), não 
produzindo uremia, mas com potente efeito emético ${ }^{5}$. Vale ressaltar, que as drogas antieméticas clássicas (metoclopramida, por exemplo) que podem antagonizar a ação da apomorfina, por terem ação antidopaminérgica central, pioram os sintomas parkinsonianos acentuadamente, e por isso não devem ser usadas. $\mathrm{O}$ aparecimento do domperidone foi a maneira de contornar este problema, porque esta droga tem ação antidopaminérgica e não ultrapassa a barreira $\mathrm{BHE}^{4}$. Os efeitos colaterais das drogas agonistas dopaminérgicas são determinados, principalmente, pela estimulação de receptores dopaminérgicos localizados fora da $\mathrm{BHE}$, como a área postrema, eminência medial e pituitária, e mesmo órgãos periféricos, como o estômago. Corsini e col., em 1979, foram os primeiros a administrarem o domperidone para contornar o efeito emético da apomorfina, abrindo, assim, uma grande perspectiva para o tratamento da doença de Parkinson 4.

A partir de 1987, começaram a surgir os relatos da experiência do uso de apomorfina (combinado ao domperidone) em pacientes parkinsonianos com FM decorrente da terapêutica com levodopa, passando a ser uma alternativa eficaz para o manejo destas complicaçōes ${ }^{13,28}$.

Nosso objetivo é o de relatar a experiência inicial com o uso da apomorfina em pacientes parkinsonianos com flutuaçōes de rendimento da levodopa.

\section{MATERIAL E MÉTODOS}

Avaliamos a resposta clínica à apomorfina em 4 pacientes atendidos no Ambulatório do Setor de Investigação em Moléstias Extrapiramidais da Disciplina de Neurologia da Escola Paulista de Medicina (EPM) com o diagnóstico de doença de Parkinson com FM secundárias ao uso de levodopa.

Os pacientes foram avaliados do ponto de vista motor de 3 formas diferentes:

-Itens 18-31 do exame motor da Escala Unificada para Avaliação de Doença de Parkinson (UPDRS), cujo escore máximo é 108 (quanto maior o escore, maior o grau de acometimento pela doença) ${ }^{11}$;

-"Tapping test", que se constitui no número de vezes em que a paciente toca a ponta do dedo indicador em dois pontos separados 20 centímetros um do outro, em 60 segundos $^{25}$; neste estudo, escolhemos o indicador da mão mais acometida e comparamos antes e depois da apomorfina; $e$

-Tempo requerido para o paciente levantar de um cadeira sem apoio para os braços, andar 6 metros, voltar e sentar novamente 25 .

Utilizamos a forma injetável de apomorfina (Apomorphine Woelm®, Munique, Alemanha) em ampolas de $1 \mathrm{ml}$ ( $10 \mathrm{mg} / \mathrm{ml})$. Domperidone $20 \mathrm{mg}$ três vezes ao dia, foi iniciado 48 horas antes da aplicação da apomorfina, exceto no Paciente 4, que recebeu simultaneamente à apomorfina.

Como a apomorfina nāo é medicamento disponível para uso clínico no Brasil, submetemos o protocolo deste estudo à aprovação da Comissão de Ética do Hospital São Paulo/EPM. Os pacientes selecionados para receberem a apomorfina foram informados previamente dos possíveis efeitos desejados e adversos da droga e os que concordaram em recebê-la foram orientados a permanecer uma noite (mínimo de 8 horas) sem receber levodopa. Na manhã seguinte, foram submetidos a avaliação motora conforme os 3 itens previamente citados. Em seguida, receberam uma dose de 1 ou $2 \mathrm{mg}$ de apomorfina por injeçāo subcutânea e, caso esta dose fosse insuficiente para produzir um estado "on", doses sucessivas de $1 \mathrm{mg}$, a cada 15 minutos, foram aplicadas até que ocorresse uma inequívoca resposta motora. Durante o efeito da apomorfina, os escores e tempos dos testes motores foram anotados a cada 15 minutos. Após o término da ação da apomorfina os pacientes podiam receber a levodopa na mesma dose que já usavam anteriormente. Foram anotados os tempos de latência até o início do efeito e a sua duraçāo. Uma das vezes, após o término do efeito, uma segunda aplicação em dose menor foi realizada para confirmar a sua eficácia.

Segue resumo da história clínica dos 4 pacientes submetidos a apomorfina:

Paciente 1 - LM, 52 anos, sexo masculino, iniciou os sintomas aos 37 anos, tendo recebido levodopa 4 anos após. Desenvolveu discinesias de pico de dose 4 meses após a levodopa. Na mesma época iniciou flutuações motoras (wearing-off). Flutuaçōes randômicas (on-off) começaram a ocorrer 5 anos após a introduçāo da levodopa. Há periodos em que chega a ficar "off" $90 \%$ do tempo. Vem recebendo levodopa + carbidopa 250/25 1/2 compr $2 x /$ dia, levodopa + carbidopa $200 / 50$ (preparação de liberação controlada) $1 / 2$ compr $2 x /$ dia, amantadina $100 \mathrm{mg}$ $3 \mathrm{x} /$ dia e triexifenidil $2 \mathrm{mg} 2 \mathrm{x} / \mathrm{dia}$. 
Paciente 2 - JRS, 55 anos, sexo masculino, iniciou os sintomas aos 43 anos, tendo recebido levodopa 11 meses após. Desenvolveu discinesias de pico de dose e flutuaçōes motoras (wearing-off) 2 anos após a introdução da levodopa. As flutuaçōes randômicas (on-off) iniciaram-se com 4 anos de levodopaterapia. Atualmente em uso de levodopa + carbidopa $250 / 251 / 2$ compr $4 \mathrm{x} / \mathrm{dia}$, bromocriptina $10 \mathrm{mg} / \mathrm{dia}$, selegelina $10 \mathrm{mg} / \mathrm{dia}$, biperideno $4 \mathrm{mg} / \mathrm{dia}$ e clonazepan $1 \mathrm{mg} / \mathrm{dia}$.

Paciente 3 - AAD, 54 anos, sexo masculino. Primeiros sintomas da doença ocorreram aos 47 anos e começou a receber levodopa após 3 anos. Desenvolveu discinesia de pico de dose 7 meses após a introduçăo da levodopa e há 1 ano tem discinesia durante o período "on" em muitas tomadas. Desde o início notou períodos "off" durante o efeito da droga. Flutuaçōes randômicas têm ocorrido com frequência após 3 anos da dopa. Nunca teve um efeito ótimo da levodopa. Em uso de: levodopa + benserazida 200/50 1/2 compr 6x/dia, amantadina $100 \mathrm{mg} 3 \mathrm{x} /$ dia e amitriptilina $25 \mathrm{mg} 3 \mathrm{x} /$ dia.

Paciente 4 - VLLD, 49 anos, sexo feminino, iniciou a doença aos 38 anos. A levodopa foi introduzida 6 meses depois dos primeiros sintomas. Flutuações em wearing-off começaram com cerca de 1 ano de tratamento com levodopa. Discinesia começou após 2 anos e meio da levodopa e flutuações motoras em on-off com 4 anos de levodopaterapia. Em uso de levodopa + benserazida 200/50 1/8 compr 12x/dia, triexifenidil $4 \mathrm{mg} /$ dia, bromocriptina $25 \mathrm{mg} / \mathrm{dia}$, selegelina $10 \mathrm{mg} / \mathrm{dia}$.

\section{RESULTADOS}

Os Pacientes 1 e 2 receberam uma dose de $2 \mathrm{mg}$ de apomorfina e tiveram inequívoca resposta motora até no máximo 12 minutos após a aplicação. O Paciente 3 não teve resposta com 2 mg e, após 15 minutos da primeira aplicação, recebeu uma dose adicional de $1 \mathrm{mg}$, tendo respondido ap6s 15 minutos (total de 30 minutos de latência). A Paciente 4 recebeu dose inicial de $1 \mathrm{mg} e$, não apresentando resposta 15 minutos depois, foi aplicada nova dose de mais $1 \mathrm{mg}$ com resposta 5 minutos após (total de latência, 20 minutos). Nenhum dos 4 pacientes apresentou náuseas ou vômitos com a medicação, mesmo a Paciente 4 que recebeu o domperidone simultaneamente à apomorfina. Todos os 4 pacientes apresentaram bocejos como efeito colateral, junto com o início do efeito motor da droga, que desapareceram poucos minutos depois. Além disso, o Paciente 1 queixou-se de parestesia perilabial com duração de 10 minutos no meio do efeito da droga e o Paciente 3 queixou-se de mal estar indefínido nos primeiros 20 minutos do efeito da apomorfina, que desapareceu espontaneamente, mesmo antes do final do efeito motor da droga.

Tabela. Dose de apomorfina, tempo de latência, duração do efeito e mudança de escores nos 4 pacientes do estudo.

\begin{tabular}{lcccc}
\hline & Paciente 1 & Paciente 2 & Paciente 3 & Paciente 4 \\
\hline Dose de apomorfina via SC & $2 \mathrm{mg}$ & $2 \mathrm{mg}$ & $\begin{array}{c}3 \mathrm{mg} \\
(2 \mathrm{mg}+1 \mathrm{mg} \\
\text { após } 15 \mathrm{~min})\end{array}$ & $\begin{array}{c}\text { (1 mg }+1 \mathrm{mg} \\
\text { após } 15 \mathrm{~min})\end{array}$ \\
$\begin{array}{l}\text { Latência do efeito } \\
\text { (em minutos) }\end{array}$ & 10 & 7 & $30(15+15)$ & $20(15+5)$ \\
$\begin{array}{l}\text { Duraçāo do efeito } \\
\text { (em minutos) }\end{array}$ & 75 & 60 & 80 & 85 \\
$\begin{array}{l}\text { UPDRS - escore } \\
\text { (antes/depois) }\end{array}$ & $57 / 27$ & $64 / 36$ & $70 / 48$ & $65 / 30$ \\
$\begin{array}{l}\text { "Taping test" - n' de toques } \\
\text { (antes/depois) }\end{array}$ & $33 / 50$ & $31 / 61$ & $59 / 95$ & $65 / 74$ \\
$\begin{array}{l}\text { Levantar de cadeiras - } \\
\text { em segundos } \\
\text { (antes/depois) }\end{array}$ & $40 / 15$ & $310 / 60$ & $45 / 22$ & $120 / 20$ \\
\hline
\end{tabular}


O efeito terapêutico obtido com a aplicação de apomorfina produziu uma fase "on" com o mesmo padrão obtido com uma dose de levodopa. Todos os pacientes apresentavam discinesia medicamentosa na fase "on" e a manifestaram durante o efeito da apomorfina. Os quatro pacientes se encontravam no estágio 4 de Hoehn-Yahr na fase "off" e no estágio 3 na fase "on".

No Paciente 1 foi aplicada nova dose de $1,5 \mathrm{mg}$ de apomorfina após o final do efeito da primeira aplicação. Nesta segunda aplicação a resposta clínica foi semelhante à obtida antes com 2 mg. A latência foi de 12 minutos e a duração do efeito, 80 minutos.

Na Tabela são mostrados os resultados obtidos com a ministração de apomorfina aos pacientes.

\section{COMENTÁRIOS}

A administraçăo de apomorfina a quatro pacientes mostrou-nos que este é um procedimento simples, sem efeitos adversos significativos, que surtiu efeito em todos. $\mathrm{O}$ estado "on" foi obtido com doses entre 1,5 e $3 \mathrm{mg}$ por aplicação subcutânea.

A apomorfina tem sido utilizada em paises europeus e mostrou que pode reduzir, nos parkinsonianos com flutuação do rendimento da levodopa, os períodos “off" em mais de $50 \%$ 12,26,27. A magnitude e o padrão da resposta clínica da apomorfina administrada por via subcutânea são virtualmente idênticos aos da levodopa. A única diferença entre as duas drogas é que o tempo de ação da apomorfina é mais curto. Segundo Kempster e col., a resposta motora à apomorfina tem duração média de $56 \mathrm{~min}$, comparada à duração média de $211 \mathrm{~min}$ para a levodopa ${ }^{20}$. A duração do efeito em nossos pacientes foi semelhante à já observada anteriormente ${ }^{12,20,26,27}$.

A resposta motora à apomorfina usualmente está presente mesmo nos pacientes com períodos “off" resistentes à levodopa. A previsibilidade de resposta da apomorfina na DP é tal, que tem sido proposto seu uso para se testar a responsividade dopaminérgica. Nesse teste, os pacientes que não respondessem à apomorfina na realidade não teriam a DP idiopática ${ }^{1}$.

A aplicação sucessiva da apomorfina em um mesmo paciente em intervalos de tempo curtos (por volta de 1 hora) não parece induzir a diminuição da magnitude da resposta subsequente à droga, ou seja, não leva ao fenômeno de tolerância ${ }^{15,18}$. Entretanto, Grandas e col. mostraram que a duração da resposta motora é menor nos pacientes que recebem a segunda dose depois de 2 horas da primeira, quando comparada à dos pacientes que receberem a segunda dose depois de 4 horas ${ }^{16}$. O uso a longo prazo da apomorfina também não parece induzir tolerância ${ }^{17}$.

A apomorfina é habitualmente administrada por via subcutânea (SC), seja em aplicaçōes intermitentes ou em infusão contínua por meio de bomba. As duas formas apresentam resultados semelhantes, mas a infusão contínua parece ser um pouco mais cômoda para o paciente, embora as doses tenham que ser maiores ${ }^{12.21,26}$. A administração de apomorfina por via sublingual (SL) tem sido utilizada mais recentemente ${ }^{19}$. Esta forma de administração produz resposta motora comparável à da via SC, embora a latência de início da ação seja maior - entre 20 e $40 \mathrm{~min}$, contra média de 14 min para a via SC, e a duração da resposta motora seja maior - até 118 min para a SL, contra média de 60 min para a $S^{8,14,19}$. Outra possibilidade é a ministração da apomorfina por via intranasal, mas ainda é pequena a experiência com esta via de administração ${ }^{22}$.

As doses de apomorfina de 1 ou $2 \mathrm{mg}$ utilizadas neste estudo basearam-se nas doses minimamente efetivas observadas em outros ensaios clínicos ${ }^{12.27}$. Estas doses variam de acordo com a via de administração. Na injeção SC intermitente, as doses variam de 1 a $5 \mathrm{mg}$ (em média $3 \mathrm{mg}$ ) de 2 a 5 vezes ao dia (em média $10 \mathrm{mg}$ ao dia) ${ }^{12,27}$. Na via SC por infusão contínua, a dose oscila de 2 a $7 \mathrm{mg}$ por hora, podendo chegar até $160 \mathrm{mg}$ ao dia ${ }^{12.26}$. Na via SL, as doses variam em torno de 40 a $60 \mathrm{mg}$, sendo administradas de 3 a 6 vezes ao dia ${ }^{8,19}$. Por via nasal, as doses variam entre 4 e $6 \mathrm{mg}$ em cada aplicação ${ }^{22}$. 
Os principais efeitos adversos da apomorfina são náuseas, vômitos e hipotensão postural, desde que o domperidone não seja administrado associadamente 4 . Uma parcela razoável de pacientes pode apresentar sonolência e bocejos nas primeiras tomadas, mas estes efeitos vão desaparecendo com o tempo ${ }^{12}$. O aparecimento de alucinações visuais pode acompanhar algumas tomadas em aproximadamente $10 \%$ dos pacientes ${ }^{12,27}$. Nódulos subcutâneos, às vezes associados a prurido, podem aparecer nos locais de aplicação da droga na via SC contínua, mas não apresentam gravidade que justifique a suspensão da droga ${ }^{12}$. Eosinofilia tem sido descrita em alguns casos (pode chegar a $10 \%$ dos pacientes no início do tratamento), mas esta é reversível após um ano, mesmo sem a suspensão da droga $a^{12,27}$. $\mathrm{Na}$ administração $\mathrm{SL}$, tem sido descrito gosto desagradável ${ }^{19}$ e estomatite em alguns pacientes ${ }^{\mathrm{n}}$.

Nossa experiência inicial com a aplicação de apomorfina é altamente satisfatória, pois um bom efeito foi conseguido com doses relativamente baixas e com poucos efeitos colaterais. Além disso, o custo relativamente baixo da droga coloca a apomorfina como uma alternativa viável para algumas fases da DP. É certo, porém, que a apomorfina nāo representa uma soluçāo definitiva para as complicações do tratamento da DP, mas seu uso criterioso pode transformar a qualidade de vida de muitos pacientes, hoje sem muitas perspectivas.

\section{REFERENCIAS}

1. Bonuccelli U, Piccini P, Del Dotto P, Rossi G, Corsini GU, Muratorio A. Apomorphine test for dopaminergic responsiveness: a dose assesment study. Mov Disord 1993,8:158-164.

2. Bravi D, Mouradian MM, Roberts JW, Davis TL, Sohn YH, Chase TN. Wearing-off fluctuations in Parkinson's disease: contribution of postsynaptic mechanisms. Ann Neurol 1994,36:27-31.

3. Clough CG. Parkinson's disease: management. Lancet 1991,337:1324-1327.

4. Corsini GU, Del Zompo M, Gessa GL, Mangoni A. Therapeutic efficacy of apomorphine combined with an extracerebral inhibitor of dopamine receptors in Parkinson's disease. Lancet 1979,1:954-956.

5. Cotzias GC, Papavasiliou PS, Fehling C, Kaufman B, Mena I. Similarities between neurologic effects of Ldopa and of apomorphine. N Engl J Med 1970,282:31-33.

6. Cotzias GC, Papavasiliou PS, Tolosa ES, Mendez JS, Bel-Midura M. Treatment of Parkinson's disease with aporphine: possible role of growth hormone. N Engl J Med 1976,294:567-572.

7. Cotzias GC, Van Woert MH, Schiffer LM. Aromatic amino acids and modifization of parkinsonism. $\mathrm{N}$ Engl J Med 1967,276:374-378.

8. Deffond D, Durif F, Toumilhac M. Apomorphine in treatment of Parkinson's disease: comparison between subcutancous and sublingual routes. J Neurol Neurosurg Psychiatry 1993,56:101-103.

9. Fabbrini G, Mouradian MM, Juncos JL, Schlegel J, Mohr E, Chase TN. Motor fluctuation in Parkinson's disease: central pathophysiological mechanisms, Part I. Ann Neurol 1988,24:366-371.

10. Fahn S. "On-off" phenomenon with levodopa therapy in parkinsonism. Neurology 1974, 24:431-441.

11. Fahn S, Elton RL, and members of the UPDRS Development Committee. Unified Parkinson's disease rating scale. In:Fahn S, Marsden CD, Calne DB, Goldstein M (eds). Recent developments in Parkinson's disease, vol 2. Florahm Park-NJ: MacMillan Health Care Information 1987:153-164.

12. Frankel JP, Lees AJ, Kempster PA, Stern GM. Subcutaneous apmorphine in the treatment of Parkinson's disease. J Neurol Neurosurg Psychiatry 1990,53:96-101.

13. Gancher ST, Nutt JG. Diumal responsiveness to apomorphine. Neurology 1987,37:1250-1253.

14. Gancher ST, Nutt JG, Woodward WR. Absorption of apomorphine by various routes in parkinsonism. Mov Disord 1991,6:212-216.

15. Gervason CL, Pollak PR, Limousin P, Perret JE. Reproducibility of motor effects induced by successive subcutaneous apomorphine injections in Parkinson's disease. Clin Neuropharmacol 1993,16:113-119.

16. Grandas F, Gancher S, Lera G, Rodriguez M, Woodward WR, Nutt J, Obeso JA. Time interval between repeated injections conditions the duration of motor improvement to apomorphine in Parkinson's disease. Neurology 1992,42:1287-1290.

17. Hughes AJ, Bishop S, Kleedorfer B, Turjanski N, Fernandez W, Lees AJ, Stern GM. Subcutaneous apomorphine in Parkinson's disease: response to chronic administration for up to five years. Mov Disord 1993,8:165-170.

18. Hughes AJ, Lees AJ, Stern GM. The motor response to sequential apomorphine in parkinsonian fluctuations. J Neurol Neurosurg Psychiatry 1991,54:358-360. 
19. Hughes AJ, Webster R, Bovingdon M, Lees AJ, Stern GM. Sublingual apomorphine in the treatment of Parkinson's disease complicated by motor fluctuations. Clin Neuropharmacol 1991,6:556-561.

20. Kempster PA, Frankel JP, Stern GM, Lees AJ. Comparison of motor response to apomorphine and levodopa in Parkinson's disease. J Neurol Neurosurg Psychiatry 1990,53:1004-1007.

21. Kempster PA, Iansek R, Larmour I. Intermitent subcutaneous apomorphine injection treatment for parkinsonian motor oscillations. Aust NZ J Med 1991,21:314-318.

22. Kleedorfer B, Turjanski N, Ryan R, Lees AJ, Milroy C, Stern GM. Intranasal apomorphine in Parkinson's disease. Neurology 1991,41:761-762.

23. Marsden CD, Parkes JD. Success and problems of long-term levodopa therapy in Parkinson's disease. Lancet 1977,1:345-349.

24. Mouradian MM, Juncos JL, Fabbrini G, Schlegel J, Bartko JJ, Chase TN. Motor fluctuation in Parkinson's disease: central pathophysiological mechanisms, Part II. Ann Neurol 1988,24:372-378.

25. Nutt JG, Woodward WR, Hammerstad JP, Carter JH, Anderson JL. The "on-off" phenomenon in Parkinson's disease: relation to levodopa absortion and transport. N Engl J Med 1984,310:483-488.

26. Poewe W, Kleedorfer B, Wagner M, Bösch S, Schelosky L. Continuous subcutaneous apomorphine infusions for fluctuating Parkinson's disease. Adv Neurol 1993,60:656-659.

27. Steiger MJ, Quinn NP, Marsden CD. The clinical use of apomorphine in Parkinson's disease. J Neurol 1992,239:389-393.

28. Stibe CMH, Lees AJ, Kempster PA, Stern GM. Subcutaneous apomorphine in parkinsonian on-off oscillations. Lancet 1988,1:403-406.

29. Tolosa ES, Martin WE, Cohen HP, Jacobson RL. Patterns of clinical response and plasma dopa in Parkinson's disease. Neurology 1975,25:177-183. 